\title{
Magnetism in Disordered Graphene and Irradiated Graphite
}

\author{
Oleg V. Yazyev* \\ Ecole Polytechnique Fédérale de Lausanne (EPFL), Institute of Theoretical Physics (ITP) \\ and Institute of Chemical Sciences and Engineering (ISIC), CH-1015 Lausanne, Switzerland and \\ Institut Romand de Recherche Numérique en Physique des Matériaux (IRRMA), CH-1015 Lausanne, Switzerland
}

(Dated: October 22, 2018)

\begin{abstract}
The magnetic properties of disordered graphene and irradiated graphite are systematically studied using a combination of mean-field Hubbard model and first principles calculations. By considering large-scale disordered models of graphene I conclude that only single-atom defects can induce ferromagnetism in graphene-based materials. Preserved stacking order of graphene layers is shown to be another necessary condition for achieving a finite net magnetic moment of irradiated graphite. $A b$ initio calculations of hydrogen binding and diffusion and of interstitial-vacancy recombination further confirm the crucial role of stacking order in $\pi$-electron ferromagnetism of proton bombarded graphite.
\end{abstract}

PACS numbers: $61.72 . \mathrm{Ji}, 61.80 . \mathrm{Az}, 75.75 .+\mathrm{a}, 81.05 . \mathrm{Uw}$

Graphene and related nanostructured materials are recognized as possible building blocks for prospective electronics technologies [1] among which spintronics [2] occupy a special place. Extraordinary magnetic properties of carbon nanostructures resulting from their reduced dimensions have been predicted theoretically [3, 4, [5]. This progress allowed to think of novel devices realizing spintronics in practice [6]. Although most of the predicted properties are still awaiting their experimental confirmation, a discovery of room-temperature ferromagnetism in proton irradiated graphite [7] continues to stimulate research in the field of magnetic carbon.

Recent experimental investigations have revealed that the magnetic order in proton bombarded graphite has two-dimensional (i.e. graphene-like) character [8] and originates from the carbon $\pi$-electron system rather than from intrinsic or introduced impurities [9]. These results are supported by theoretical calculations [10, 11] which show the crucial role of defect-induced quasilocalized states [12, 13] in producing a magnetic order stable at high temperatures. Although magnetic properties of defects in graphene have been widely studied by first principles methods, the use of different periodic models of limited size often resulted in varying outcomes [10, 14, 15, 16, 17]. This calls for a study employing realistic disordered models. The origin of ferromagnetic order in graphene which generally favors antiferromagnetic coupling between the neighboring atoms [4, 10] as well as the role of various possible defects in inducing magnetism remain elusive.

In this Letter, I address magnetic properties of disordered graphene and irradiated graphite by using a combination of mean-field Hubbard model and first principles calculations. The Hubbard model calculations of realistic models of disordered graphene show that only singleatom defects such as vacancies or chemical functionalizations unequally distributed over the two sublattices may result in a net magnetic moment. Further first principles calculations point out that the stacking order of graphite lies at the origin of ferromagnetism in proton bombarded graphite.

To study the magnetic properties of disordered graphene I employ the mean-field approximation of the Hubbard model which proved to describe carbon $\pi$ electron systems in good agreement with first principles calculations [5]. The corresponding Hamiltonian reads

$$
\mathcal{H}=-t \sum_{\langle i, j\rangle, \sigma}\left[c_{i \sigma}^{\dagger} c_{j \sigma}+\text { h.c. }\right]+U \sum_{i, \sigma} n_{i \sigma}\left\langle n_{i-\sigma}\right\rangle,
$$

where the first part is the single-orbital tight-binding Hamiltonian while the second part accounts for the onsite Coulomb repulsion. In this expression $c_{i \sigma}\left(c_{i \sigma}^{\dagger}\right)$ annihilates (creates) an electron with spin $\sigma$ at site $i$ and $\langle i, j\rangle$ stands for the pairs of bonded atoms. The expectation values of the spin-resolved density $n_{i \sigma}=c_{i \sigma}^{\dagger} c_{i \sigma}$ on atom $i$ are obtained from the eigenvectors of $\mathcal{H}$. The self-consistent solution of the problem provides the spin densities $M_{i}=\left(n_{i \uparrow}-n_{i \downarrow}\right) / 2$ on each atom $i$.

The models of disordered graphene are generated by distributing randomly point defects in a large $20 \times 20$ supercell (800 atoms). The calculations are performed for 64 different random distributions in order to provide reliable statistical sampling. This computational protocol yields the mean magnetic moments converged within $\sim 1 \%$ both with respect to the supercell size and the number of configurations. Similar approach was successfully applied to study vacancy-induced magnetism in oxide materials [18]. I consider the range of defect concentration $x=N_{\mathrm{d}} / N=0.01-0.1$ with $N_{\mathrm{d}}$ out of $N$ atoms or bonds being affected. The values $U / t=1.0,1.33,1.67,2.0$ are studied in order to investigate the role of on-site Coulomb repulsion magnitude. While the value of hopping integral $t \approx 2.7 \mathrm{eV}$ is well established for graphene, there is a growing debate regarding the on-site Coulomb repulsion $U$. It is worth pointing out that the magnitude of $U$ was inferred from 
magnetic resonance studies of neutral soliton states in trans-polyacetylene [19], a one-dimensional bipartite $s p^{2}$ carbon system closely related to graphene. The estimated $U=3.0-3.5 \mathrm{eV}(U / t=1.1-1.3)$ [20] closely corresponds to $U / t=1.33$ considered here.

The empirical value of $U / t$ can also be compared to the values obtained by establishing a relation between the results of first principles and mean-field Hubbard model calculations 21]. The local density approximation (LDA) leads to $U / t \approx 0.9$ while the generalized gradient approximation (GGA) gives $U / t \approx 1.3$ in good agreement with the empirical value. It is somewhat cautionary that the results provided by generally more accurate hybrid density functionals correspond to $U / t \approx 2.0$ which is very close to the critical value $U / t \approx 2.2$ at which graphene accepts an antiferromagnetic ground state 22]. Discrepancies between the results of hybrid density functional calculations and experimental data for trans-polyacetylene have also been reported [23]. This urges to reconsider performance of these methods for describing magnetic $s p^{2}$ carbon systems.

Disorder in graphene induced by chemical treatment or irradiation with ions or electrons can be conveniently classified by the following three types of point defects. A carbon atom removed from the $s p^{2}$ lattice (i) ("vacancy $\left.^{6}\right)$ corresponds to either true vacancy [10, 14] or the rehybridization into the $s p^{3}$ configuration. Such rehybridization may result from chemical functionalization (e.g. hydrogen chemisorption [10, 24]) or binding of the interstitial carbon atoms in irradiated graphite [25, 26]. A multiple-atom 'vacancy' can be viewed as an ensemble of neighboring single-atom 'vacancies'. In some cases a bivalent chemical functionalization results in breaking the bond between the pairs of adjacent atoms without their rehybridization [27]. This leads to so-called bond dilution (ii). While (i) and (ii) give rise to undercoordinated carbon atoms, topological defects (iii) such as the Stone-Wales defect 28] maintain the coordination number. This type of disorder can be described as a permutation of connectivity involving two or more neighboring atoms. However, no magnetic moments due to the presence of the Stone-Wales defects were observed in this study as well as in previous ab initio calculations [15]. Below, I focus only on the magnetism induced by the 'vacancies' and the bond dilution disorder.

The Hubbard model solutions for all configurations considered are characterized by the antiparallel orientation of magnetic moments on the two sublattices. Figs. 1 (a,b) show the sublattice-resolved average magnetic moments $\left\langle M_{i}^{\eta}\right\rangle(\eta=A, B)$ induced by varying concentrations of "vacancies' for different values of $U / t$. For the case of defects equally distributed over the two sublattices [Fig. 1(a)] $\left\langle M_{i}^{\mathrm{A}}\right\rangle$ and $\left\langle M_{i}^{\mathrm{B}}\right\rangle$ compensate each other resulting in an antiferromagnetic state with zero total magnetic moment $M=N\left(\left\langle M_{i}^{\mathrm{A}}\right\rangle+\left\langle M_{i}^{\mathrm{B}}\right\rangle\right) / 2=0$. This result can be understood within the Lieb theorem
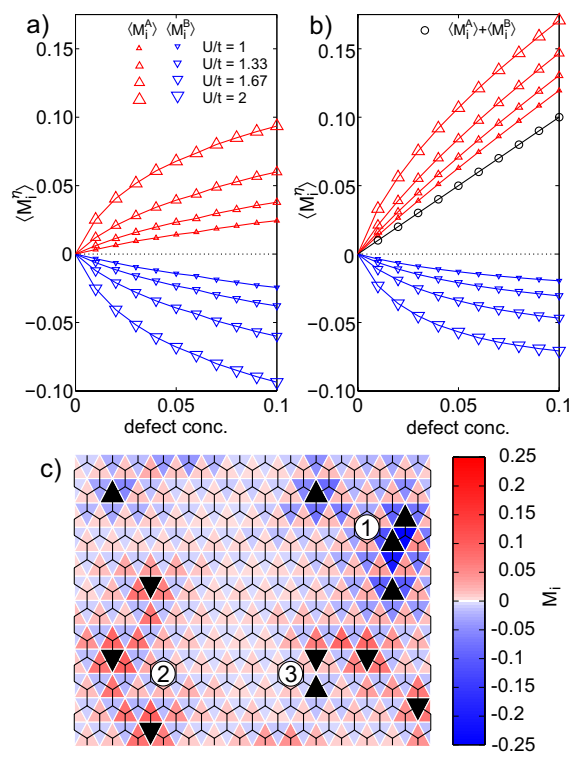

FIG. 1: (Color online) Average magnetic moments of carbon atoms in $A$ and $B$ sublattices of graphene as a function of "vacancy' concentration for different $U / t$ values. The "vacancies" are either (a) distributed equally among sublattices or (b) belong to sublattice $B$ only. (c) Spin density distribution in a representative configuration with 'vacancy' defects $(x=0.03)$ in sublattices $A(\mathbf{\Lambda})$ and $B(\mathbf{\nabla})$.

29] which relates the total magnetic moment of a halffilled bipartite system to the difference of number of atoms in the two sublattices. In our case the two sublattices contain equal number of atoms resulting in zero net magnetic moment. The sublattice-resolved magnetic moments $\left\langle M_{i}^{\eta}\right\rangle \propto x^{\gamma}(0<\gamma<1)$ show sub-linear dependence on $x$ and tend to increase with increasing the magnitude of $U / t$. In particular, for $U / t=1.33$ the dependence on 'vacancy' concentration $x$ can be accurately fitted by $\left|\left\langle M_{i}^{\eta}\right\rangle\right| \approx 0.234 \cdot x^{0.78}$. For the case of 'vacancies' in sublattice $B$ only the total magnetic moment per unit cell $M=x$ [open circles in Fig. 1(b)]. This is again in strict agreement with the Lieb theorem predicting $2 M=N_{\mathrm{A}}-N_{\mathrm{B}}$. The magnetic state originates from the Hund's rule population of $N_{\mathrm{A}}-N_{\mathrm{B}}$ zero-energy defect-induced quasilocalized states in sublattice $A$. However, these singly-occupied states induce exchange polarization of the fully-occupied ones leading to a significant antiparallel magnetization of the atoms in sublattice $B$. The induced magnetic moments follow an approximate dependence $\left\langle M_{i}^{\mathrm{B}}\right\rangle \approx-0.159 \cdot x^{0.69}$.

Figure1(c) illustrates the distribution of magnetic moments induced by the "vacancy' disorder in a representative configuration $(x=0.03)$ with defects in both sublattices. Magnetic moments develop in the nanometer-size regions characterized by the local domination of "vacancies $^{6}$ in one of the sublattices [1 and $\mathbf{2}$ in Fig. 11(c)]. In these regions one can trace distinctive triangular patterns associated with individual quasilocalized states [12]. The 


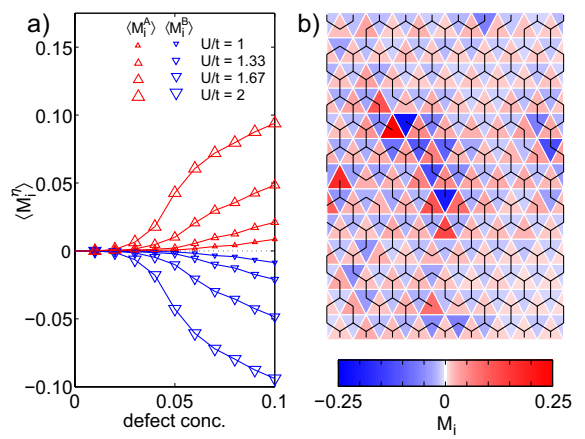

FIG. 2: (Color online) (a) Average magnetic moments of carbon atoms in $A$ and $B$ sublattices of graphene as a function of bond dilution for different $U / t$ values and (b) spin density distribution in a representative configuration $(x=0.10$; $U / t=1.33)$.

orientation of magnetic moments is determined by the antiferromagnetic coupling between the two sublattices. In contrast, the proximity of two defects pertaining to opposite sublattices [3 in Fig. 1(c)] produces no local magnetic moments. This can be explained by the fact that the quasilocalized states on different sublattices form a pair of bonding/antibonding states [11], and the energy splitting increase with decreasing the distance between the two "vacancies'. Such dispersion provides a mechanism for escaping the low-energy instability which is preferred to the spin polarization. Increased contribution of this channel with increasing defect concentration explains the sublinear dependence of induced magnetic moments on $x$ for the case of "vacancies' in both sublattices. Both mechanisms contribute to stabilizing low-energy states. Indeed, band gaps increasing from $0.14 \mathrm{eV}$ to $0.29 \mathrm{eV}$ are found in the range of concentrations $x=0.01-0.1$ and $U / t=1.33$. This agrees with the recent experimental observation of insulating behavior in proton irradiated graphite [30].

In contrast to the "vacancy" disorder, bond dilution does not change the number of atoms and, thus, can not produce a net magnetic moment. Nevertheless, antiferromagnetic correlations develop as a result of bond dilution [Fig. 2(a)]. At low values of bond dilution $(x<0.05$ for $U / t=1.33$ ) only very small average magnetic moments develop as a results of occasional occurrence of highly undercoordinated atoms. For larger values of $x$ spin polarization occurs in extended regions of higher local concentration of broken bonds as shown in Fig. 2(b) for $x=0.1$ and $U / t=1.33$. Such cumulative behavior is similar to the quantum phase transition predicted for hexagonal graphene nanoislands [5].

One can now conclude that a macroscopic magnetic moment may appear only in the presence of 'vacancy" disorder unequally distributed over the two graphene sublattices. In bulk graphite and multilayer graphene the sublattices are inequivalent due to the stacking or-

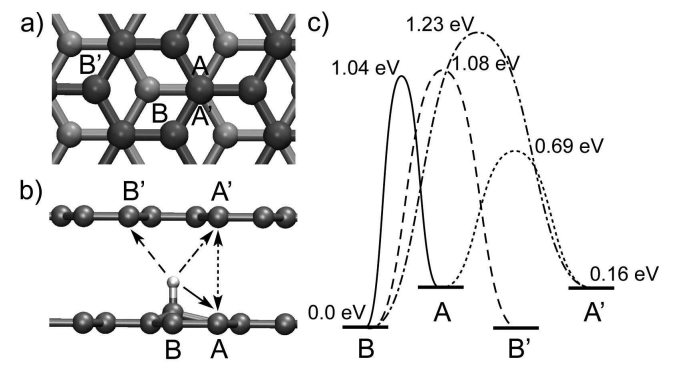

FIG. 3: (a) Inequivalent carbon atoms $(A$ and $B)$ in graphite. (b) Possible pathways for the diffusion of chemisorbed hydrogen in the $a b$ plane of graphite. (c) Relative energies of the potential energy surface minima and transition states for the diffusion of chemisorbed hydrogen in graphite.

der [Fig. 3(a)]. Irradiation of graphite with high-energy ions initially produces (i) vacancy-interstitial pairs due to knock-on recoils and (ii) chemisorbed ions stopped by the bulk material. It was found that proton bombardment of graphite leads to much stronger magnetic signal than the He-ion irradiation where no chemisorption takes place. This suggests that the hydrogen chemisortion plays an important role in the onset of magnetism in proton bombarded graphite.

The effect of stacking order in graphite is further investigated by means of first principles calculations. I considered a $4 \times 4 \times 1$ supercell of ABA stacking graphite within the GGA density functional theory framework [31, 32]. Chemisorption of a proton in position $B$ was found to be $0.16 \mathrm{eV}$ lower in energy than in position $A$ [Fig. 3(b,c)]. This is explained by the steric repulsion between the proton chemisorbed on atom $A$ and carbon atom $A^{\prime}$ of the adjacent graphene layer. A magnetic moment of $1 \mu_{B}$ localized in the functionalized graphene sheet is observed for the hydrogen chemisorption in both inequivalent positions of the crystalline lattice of graphite. This is in full agreement with the previous ab initio calculations [10, 17] and the present Hubbard model results for graphene. The barrier for the migration of proton initially trapped in position $A$ to the neighboring position $B$ is $0.88 \mathrm{eV}$ (path $A \rightarrow B$ in Fig. 3(c)). Such migration can take place at the experimental conditions. The overall barrier for the in-plane diffusion of protons in graphite (paths $A \rightarrow B, B \rightarrow B^{\prime}$ and $A^{\prime} \rightarrow B$ in Fig. 3(b,c)) defined by the lowest barrier of path $A \rightarrow B$ is $1.04 \mathrm{eV}$. Diffusion in the direction of $c$-axis requires much higher activation energy of $\sim 4 \mathrm{eV}$. Free diffusion of protons in graphite will result in either saturation of vacancy dangling bonds [14] or in the formation of energetically favorable aggregates with both sublattices being populated [17]. Magnetic moments of the hydrogen aggregates quench due to the large splitting of quasilocalized states in the complementary sublattices. In addition, the condition of preserving the stacking order of graphite limits the maximum irradiation dose and intensity. Subsequently, there exist only 
relatively narrow range of conditions at the sublatticediscriminating hydrogen chemisorption can be achieved. This accords with the fact magnetic moments are induced only in the narrow ring surrounding the irradiated spot [9].

Similarly to hydrogen chemisorption, single-atom vacancies produced by the knock-on recoils induce lowenergy quasilocalized states [10]. In contrast, interstitial carbon atoms in the energetically preferred configuration [25, 33] lead to the rehybridization of the pairs of neighboring atoms in the adjacent graphene layers. This situation is equivalent to the case of di-vacancies providing no low-energy states required for developing magnetic moments. Cross-sections for the momentum transfer due to knock-on collisions are likely to be equal for both $A$ and $B$ carbon atoms in graphite. However, the stacking order of graphite has strong influence on the kinetics of interstitial-vacancy recombination. Instantaneous recombination of low-energy recoils was found to be significantly more likely for the atoms in position $A$ [26]. At longer time scales, diffusing interstitials encounter different barriers for the recombination with vacancies in these two positions. First principles calculations show different minimum transition barriers of $0.04 \mathrm{eV}$ and $0.22 \mathrm{eV}$ for $A$ and $B$ vacancies, respectively. Both values are below the diffusion barrier of interstitial atoms $(\sim 0.5 \mathrm{eV}[34])$ suggesting that only very small difference in populations of the two sublattices by vacancy defects can be achieved in practice. Nevertheless, both vacancies and chemisorbed hydrogen atoms are found to populate preferably sublattice $B$ thus producing ferrimagnetic order with the spin polarization larger for sublattice $A$.

In conclusion, I have shown the crucial role of single-atom defects in combination with a sublatticediscriminating mechanism for developing ferromagnetic order in graphene-based materials. In graphite the role of such mechanism is played by the stacking order of graphene layers. This suggestion is confirmed by the $a b$ initio investigation of hydrogen chemisorption and vacancy defects in proton bombarded graphite. The results of this study pave a way for tailoring carbon based magnetic materials by means of irradiation and chemical treatment of graphite and other graphene derivatives.

I acknowledge the comments of P. Esquinazi, L. Helm, A. Krasheninnikov and W. L. Wang. The work was partly supported by the Swiss NSF. First principles calculations have been performed at the CSCS (Manno).

oleg.yazyev@epfl.ch

[1] P. Avouris, Z. H. Chen, and V. Perebeinos, Nat. Nanotechnol. 2, 605 (2007).

[2] I. Žutić, J. Fabian, and S. Das Sarma, Rev. Mod. Phys. 76, 323 (2004).

[3] M. Fujita, K. Wakabayashi, K. Nakada, and K. Kusak- abe, J. Phys. Soc. Jpn. 65, 1920 (1996); Y.-W. Son, M. L. Cohen, and S. G. Louie, Phys. Rev. Lett. 97, 216803 (2006); J. Fernández-Rossier, Phys. Rev. B 77, 075430 (2008).

[4] L. Brey, H. A. Fertig, and S. Das Sarma, Phys. Rev. Lett. 99, 116802 (2007).

[5] J. Fernández-Rossier and J. J. Palacios, Phys. Rev. Lett. 99, 177204 (2007).

[6] Y.-W. Son, M. L. Cohen, and S. G. Louie, Nature 444, 347 (2006); O. V. Yazyev and M. I. Katsnelson, Phys. Rev. Lett. 100, 047209 (2008); W. L. Wang, S. Meng, and E. Kaxiras, Nano Lett. 8, 241 (2008); O. V. Yazyev, W. L. Wang, S. Meng, and E. Kaxiras, Nano Lett. 8, 766 (2008).

[7] P. Esquinazi et al., Phys. Rev. Lett. 91, 227201 (2003).

[8] J. Barzola-Quiquia et al., Phys. Rev. B 76, 161403(R) (2007).

[9] H. Ohldag et al., Phys. Rev. Lett. 98, 187204 (2007).

[10] O. V. Yazyev and L. Helm, Phys. Rev. B 75, 125408 (2007).

[11] H. Kumazaki and D. S. Hirashima, J. Phys. Soc. Jpn. 76, 064713 (2007); J. J. Palacios, J. Fernández-Rossier, and L. Brey, Phys. Rev. B 77, 195428 (2008).

[12] H. A. Mizes and J. S. Foster, Science 244, 559 (1989); K. Kelly and N. Halas, Surf. Sci. 416, L1085 (1998).

[13] V. M. Pereira et al., Phys. Rev. Lett. 96, 036801 (2006); V. M. Pereira, J. M. B. Lopes dos Santos, and A. H. Castro Neto, Phys. Rev. B 77, 115109 (2008).

[14] P. O. Lehtinen et al., Phys. Rev. Lett. 93, 187202 (2004).

[15] E. J. Duplock, M. Scheffler, and P. J. D. Lindan, Phys. Rev. Lett. 92, 225502 (2004).

[16] L. Pisani, B. Montanari, and N. M. Harrison, New J. Phys. 10, 033002 (2008).

[17] D. W. Boukhvalov, M. I. Katsnelson, and A. I. Lichtenstein, Phys. Rev. B 77, 035427 (2008).

[18] G. Bouzerar and T. Ziman, Phys. Rev. Lett. 96, 207602 (2006).

[19] W. P. Su, J. R. Schrieffer, and A. J. Heeger, Phys. Rev. Lett. 42, 1698 (1979).

[20] H. Thomann et al., Phys. Rev. B 31, 3141 (1985); S. I. Kuroda and H. Shirakawa, Phys. Rev. B 35, 9380 (1987).

[21] L. Pisani et al., Phys. Rev. B 75, 064418 (2007).

[22] N. M. R. Peres, M. A. N. Araújo, and D. Bozi, Phys. Rev. B 70, 195122 (2004).

[23] T. Bally, D. A. Hrovat, and W. T. Borden, Phys. Chem. Chem. Phys. 2, 3363 (2000).

[24] P. Ruffieux et al., Phys. Rev. Lett. 84, 4910 (2000).

[25] R. H. Telling et al., Nat. Mater. 2, 333 (2003).

[26] O. V. Yazyev et al., Phys. Rev. B 75, 115418 (2007).

[27] Y.-S. Lee and N. Marzari, Phys. Rev. Lett. 97, 116801 (2006); J. Chen et al., Science 282, 95 (1998).

[28] A. J. Stone and D. J. Wales, Chem. Phys. Lett. 128, 501 (1986).

[29] E. H. Lieb, Phys. Rev. Lett. 62, 1201 (1989); 62, 1927(E) (1989).

[30] K. Schindler, N. García, P. Esquinazi, and H. Ohldag, (unpublished).

[31] J. P. Perdew, K. Burke, and M. Ernzerhof, Phys. Rev. Lett. 77, 3865 (1996).

[32] S. Baroni et al., http://www.quantum-espresso.org/.

[33] L. Li, S. Reich, and J. Robertson, Phys. Rev. B 72, 184109 (2005).

[34] Y. Ma, Phys. Rev. B 76, 075419 (2007). 